\title{
TERCERA GENERACIÓN EN CIRUGÍA MÍNIMAMENTE INVASIVA PARA LA CORRECCIÓN DE LA INCONTINEN- CIA DE ORINA DE ESFUERZO: TVT-SECUR
}

\author{
Vicente Solà D. ${ }^{1}$, Jack Pardo S. ${ }^{1}$, Paolo Ricci $A .{ }^{1}$ \\ ${ }^{1}$ Unidad de Uroginecología y Cirugía Vaginal, Departamento de Obstetricia y Ginecología, Clínica Las Condes.
}

\begin{abstract}
RESUMEN
Objetivo: Revisar la seguridad, eficacia y principales ventajas de la corrección quirúrgica de incontinencia de orina de esfuerzo (IOE), con el nuevo dispositivo TVT-Secur. Método: Entre enero y marzo de 2007 se realizó corrección quirúrgica a 9 pacientes con incontinencia de orina de esfuerzo, a las que se les realizó urodinamia monocanal y medición de presión de retro-resistencia uretral, como parte del estudio y selección preoperatoria. Todas debían tener incontinencia por al menos un año. Se utilizó la técnica de aplicación de cinta suburetral, con el nuevo dispositivo TVT-Secur. Las medias fueron: edad 50 años, IMC 28, paridad 3 hijos e IOE 3 años (rango: 2 a 6 años). Resultados: Se realizaron 6 TVT-Secur aplicando la cinta en "U" y 3 en " $V$ ". La media de tiempo operatorio fue de 10 minutos (rango: 8 a 15 minutos). No se registraron complicaciones durante el intraoperatorio y postoperatorio inmediato, temprano y tardío. La escala visual análoga para dolor registrada a las 12 horas fue de 1 a 2. Conclusiones: La técnica de corrección de la incontinencia de orina de esfuerzo por medio de la aplicación de cinta suburetral, con el nuevo dispositivo TVT-Secur, es factible, segura y eficaz. Permite realizar una cirugía con todas las características de mínima invasión. Se trata de un sistema de cinta de tercera generación, con menor invasión que las técnicas antecesoras, ya que sólo requiere una apertura de la mucosa suburetral y no de puntos de salida por piel, dejando sólo $8 \mathrm{~cm}$ de cinta. Es un instrumento con gran versatilidad, ya que permite colocar la cinta en "U" como en la técnica de TVT o en "V" como las técnicas trans-obturadoras.
\end{abstract}

\section{PALABRAS CLAVES: Cirugía mínimamente invasiva, cinta suburetral, incontinencia de orina de esfuerzo, TVT-Secur}

\section{SUMMARY}

Objective: To evaluate the feasibility, efficacy, safety and main advantages of the stress urinary incontinence (SUI) surgical correction with the new device TVT-Secur. Method: Between January and March of 2007, surgical correction was made in 9 patients. All patients were studied with urodynamic before surgery. All must have the urinary incontinence by more of one year. The new TVT-Secur device was used. The median was: age 50 years old, BMI 28, parity 3, SUI 3 years. Results: Six TVT-Secur were applied in "U" and three in "V". The media surgical time was 10 minutes (8-15 minutes). Complications were not registered during intraoperative and immediate, early or delayed postoperative time. The visual analogue scale of pain was 1 to 2 at 12 hours. Conclusions: The new TVT-Secure technique is feasible, safe and effective in the surgical correction of the SUI. This technique allows to make a minimally invasive surgery. It is a sling system with smaller invasion than the preceding techniques, only requires an opening of the suburethral mucosa and only $8 \mathrm{~cm}$ of tape are used. It is an instrument with great versatility, since it allows to place the tape in "U" (like TVT technique) or in "V" (like trans-obturator techniques)

KEY WORDS: Minimally invasive surgery, suburethral sling, stress urinary incontinence, TVT-Secur 


\section{INTRODUCCIÓN}

A lo largo de los años, se han presentado diferentes cirugías para la corrección quirúrgica de la incontinencia de orina de esfuerzo. Una de las más exitosas han sido las que ubican una cinta en posición media suburetral, ya que ofrecen todas las ventajas de una cirugía mínimamente invasiva con un alto porcentaje de corrección. La primera de ellas fue el TVT (Trans-Vaginal-Tape) descrito en 1996 por Ulmsten $(1,2)$, cirugía que requiere de cistoscopia para comprobar la indemnidad vesical (3). Delorme en 2001describe la técnica de TOT (Trans-Obturator-Tape) $(4,5)$, que no requiere de citoscopía, posee menos riesgo de perforación de vejiga y las agujas no pasan por el espacio retropúbico (6). De esta forma se simplifica la técnica y se reduce la posibilidad de complicaciones. Más tarde, en 2003, Jean de Leval, en un intento de simplificar y hacer aún más segura la técnica, describe el TVT-O (Trans-Vaginal-Tape-Obturator) (7-9). Finalmente, en el último trimestre del 2006 se presentó una nueva técnica, de mayor simplificación, el TVT-Secur (10).

Con el objetivo de revisar la factibilidad, seguridad y eficacia del nuevo sistema TVT-Secur, analizamos en forma prospectiva nuestra experiencia con los primeros casos realizados en el país.

\section{PACIENTES Y MÉTODO}

Entre enero y marzo del 2007 se realizó un estudio prospectivo descriptivo en pacientes ingresadas para corrección quirúrgica de la incontinencia de orina de esfuerzo (IOE), en la Unidad de Uroginecología y Cirugía Vaginal de Clínica Las
Condes. Para ingresar al estudio todas las pacientes debían presentar incontinencia de orina a pequeños esfuerzo al menos por un año y de carácter permanente.

Se realizó corrección de la incontinencia de orina de esfuerzo a 9 pacientes con el nuevo sistema TVT-Secur. La edad fluctuó entre 31 y 59 años, con una mediana de 50 años. El IMC medio fue 28 (rango: 25 a 37), la paridad de 3 (rango: 2 a 4). En 5 pacientes se asoció alguna cirugía durante la misma anestesia (Tabla I).

A todas se les realizó como parte del estudio preoperatorio una urodinamia monocanal, con medición de presión de retro-resistencia uretral y cistometrograma. El tipo de IOE se clasificó según el sistema de McGuire y colaboradores (11). Seis pacientes presentaron una IOE tipo II con asociación de deficiencia intrínseca de esfínter (II+III), en los que se colocó la cinta en "U". Tres pacientes presentaron incontinencia tipo II pura, en ellas se colocó la cinta en "V".

En 5 pacientes se asoció alguna cirugía al procedimiento de TVT-Secur. En todas ellas se realizó primero la cirugía agregada y luego la cirugía anti-incontinencia. Se realizaron 4 histerectomías por miomas sintomáticos ( 3 totales laparoscópicas y 1 laparoscópica asistida vaginal), a una de ellas también se asoció una malla Prolift (12) para la corrección de un cistocele grado III. La quinta paciente en que se asoció otra cirugía, se realizó una colpoperineoplastía por amplitud vaginal sintomática (13).

El dispositivo TVT-Secur está formado por dos ramas que en su extremo anterior soportan una malla de polipropileno macroporo monofilamento (idéntica a la de TVT clásico). La malla en el lugar

Tabla I

CASOS INGRESADOS AL ESTUDIO

\begin{tabular}{lcclcccc}
\hline Caso & Edad & $\begin{array}{c}\text { Urodinamia } \\
\text { monocanal }\end{array}$ & Diagnóstico asociado & TVT-S (Tipo) & $\begin{array}{c}\text { Cirugía } \\
\text { asociada }\end{array}$ & Cistoscopia & EVA 12 $h$ \\
\hline 1 & 46 & II+III & Miomas sintomáticos & U & HTL & Sí & 3 \\
2 & 59 & II & Ninguno & V & No & No & 1 \\
3 & 52 & II+III & Ninguno & U & No & Sí & 1 \\
4 & 52 & II+III & Ninguno & No & Sí & 2 \\
5 & 50 & II & Miomas+Cistocele grado III & V & HTLAV+PA & No & 4 \\
6 & 52 & II+III & Ninguno & U & NO & Sí & 1 \\
7 & 42 & II+III & Miomas sintomáticos & U & HTL & Sí & 3 \\
8 & 31 & II & Amplitud vaginal sintomática & V & CAVS & No & 3 \\
9 & 46 & II+III & Miomas sintomáticos & U & HTL & Sí & 3 \\
\hline
\end{tabular}

HTL: Histerectomía total laparoscópica; HTLAV: histerectomía total laparoscópica asistida por vía vaginal; CAVS: colpoperineoplastía por amplitud vaginal sintomática; PA: prolift anterior; EVA: escala visual análoga del dolor. 
de sujeción a las ramas, se encuentra a manera de sándwich encerrada por dos láminas de material de fijación absorvible, vicryl (poliglactin 910) y sutura de PDS (polidioxanon). En el extremo posterior posee un sistema que permite soltar la malla una vez puesta bajo la uretra media, a través de la tracción de una prolongación metálica. La rama derecha en su extremo proximal (el de la malla) posee una cubierta protectora que permite identificarla (Figura 1). Este dispositivo permite colocar la malla bajo la uretra en " $\mathrm{V}$ " (como en el TOT o el TVT-O) o en "U" (como en el TVT).

Se utilizó anestesia general o espinal en los casos en que hubo cirugía laparoscópica o solo vaginal, respectivamente.

Técnica quirúrgica:

Paso 1: bajo anestesia se coloca la paciente en posición ginecológica en 90 grados de flexión muslo-abdomen.

Paso 2: se instala sonda Foley 16 o 18 FR, permitiendo un vaciamiento completo de la vejiga.

Paso 3: se realiza una incisión longitudinal de $12 \mathrm{~mm}$ en la mucosa vaginal, a nivel de tercio medio uretral (Figura 2).

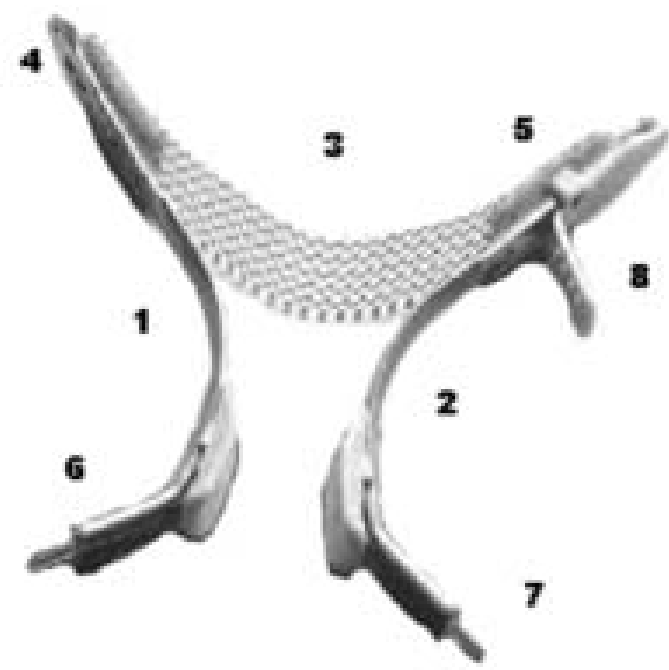

Figura 1. Dispositivo TVT-Secur. Dispositivo TVT-Secur: formado por dos ramas (1-2) que en su extremo anterior soportan una malla de polipropileno macroporo monofilamento (3). La malla en el lugar de sujeción de las ramas, se encuentra a manera de sándwich encerrada por dos láminas de material de fijación absorvible, vicryl (poliglactin 910) y sutura de PDS (polidioxanon) (45). En el otro extremo (posterior) posee un sistema que permite soltar la malla una vez puesta bajo la uretra media, a través de la tracción de una prolongación metálica (6-7). La rama derecha en su extremo proximal (el de la malla) posee una cubierta protectora que permite identificarla (8).

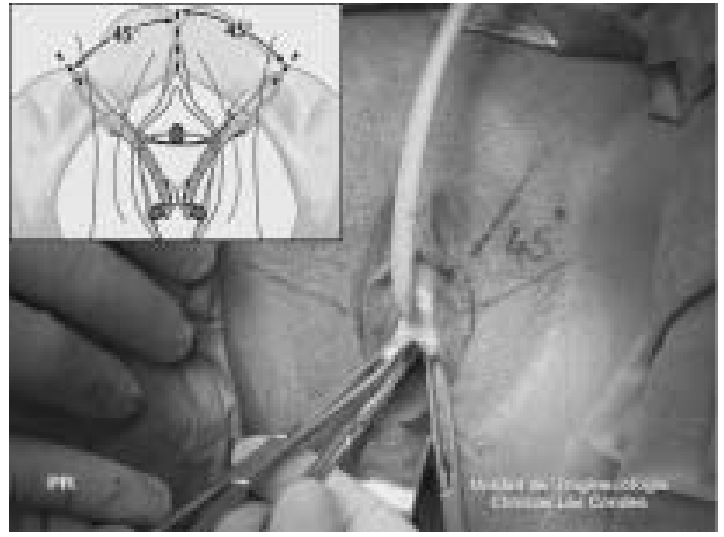

Figura 2. Apertura de mucosa suburetral. En piel se dibujan lo dos posibles trayectos para aplicar la cinta, en "U" como la técnica de TVT o en "V" como las transobturadoras.

Paso 4: si se opta por la colocación en "U", se realiza una disección en 45 grados de 2 a $2,5 \mathrm{~cm}$ de longitud, respecto a la línea media, con tijeras de Metzenbaun en el tejido parauretral (Figura 3). Si se opta por colocación en "V" se realiza una disección en más de 45 grados, también de 2 a $2,5 \mathrm{~cm}$ de longitud.

Paso 5: si se opta por la colocación en "U", se debe colocar un movilizador vesical dentro de una sonda Foley para desplazar la vejiga contra lateralmente al lado donde se inserta la rama del dispositivo.

Paso 6: se toma la rama del dispositivo TVTSecur que se va a introducir, con un porta aguja, por su extremo distal y se introduce en el espacio disecado. Se repite la maniobra con la rama contra lateral, ajustando la cinta de polipropileno bajo

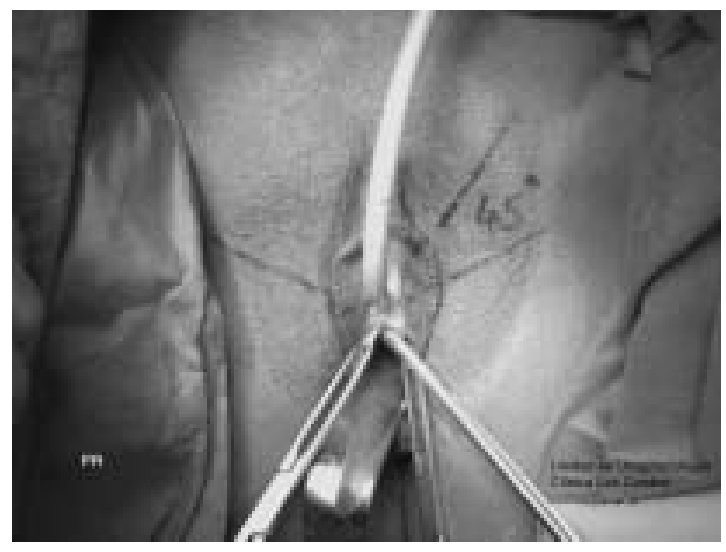

Figura 3. Apertura parauretral con tijera. 
la uretra media, siempre con técnica libre de tensión (Figura 4 y 5).

Paso 7: si se ha optado por dejar la cinta en "U", se debe realizar una cistoscopia de revisión para comprobar indemnidad vesico-uretral, al igual que en la técnica de TVT.

Paso 8: se retira el mango de inserción de cada lado con un pequeño movimiento de rotación y tracción, empujando el extremo de fijación del dispositivo hacia las estructuras óseas de la pelvis. En caso necesario, antes de retirar ambos mangos se puede volver a rectificar el ajuste, por medio de la tracción de las ramas y/o con una tijera de Metzembaum (Figura 6).

Paso 9: se realiza colporrafia con vicryl 3-0 corrido.

Si se opta por la colocación de la cinta suburetral en "Hammock" se puede prescindir del movilizador vesical y de la cistoscopia de revisión. La sonda vesical se retiró 1 a 2 horas en los caso de TVT-Secur sin cirugía asociada y en los casos con histerectomía se retiró a las 12 horas. $\mathrm{La}$ analgesia utilizada fue ketoprofeno $100 \mathrm{mg}$ endovenoso cada 8 horas, por tres dosis, luego ibuprofeno $600 \mathrm{mg}$ cada 12 horas vía oral, por 7 días. La levantada y deambulación se indicó entre 4 y 6 horas post cirugía. El alta se efectuó a las 12 horas en los casos de TVT-Secur, después de verificar dos micciones espontáneas. En todas las pacientes se registró el dolor mediante escala visual análoga del dolor (EVA) a las 12 horas de postoperatorio.

Todas las pacientes fueron controladas ambulatoriamente a los siete días. Durante este control se preguntó el grado de satisfacción con la cirugía y los resultados obtenidos.

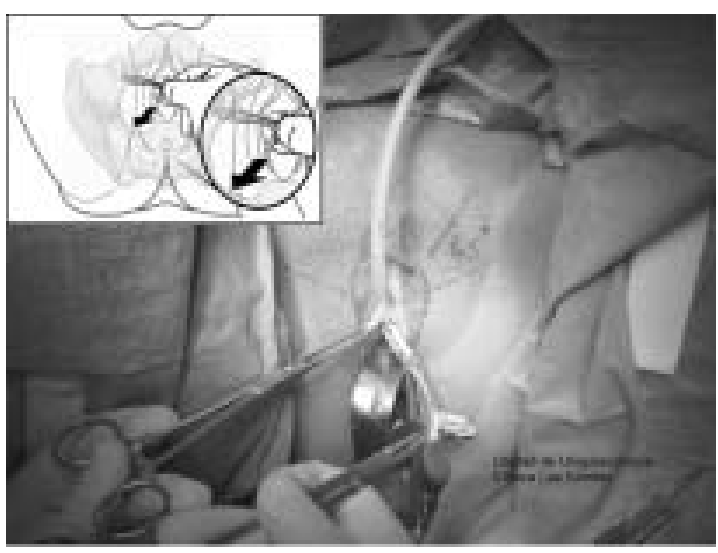

Figura 4. Inserción de la rama izquierda del dispositivo TVT-S.

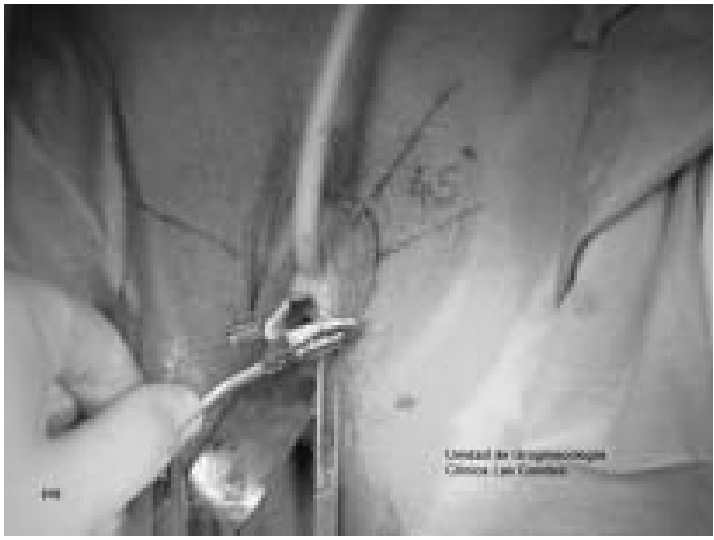

Figura 5. Inserción de la rama derecha del TVT-S. Se ha retirado de su extremo la cubierta identificadora. Se aprecia la angulación para colocar la malla en "V".

\section{RESULTADOS}

De las 9 pacientes, en 3 se colocó la malla en "V" y en 6 en "U". La media de tiempo operatorio fue de 10 minutos (rango: 8 a 15 minutos). No se registraron complicaciones en el intraoperatorio, ni el postoperatorio inmediato, temprano y tardío. En todas las pacientes se solucionó la incontinencia de orina de esfuerzo. Todas las pacientes durante el primer control ambulatorio, a los 15 días, expresaron encontrarse satisfechas con la cirugía y los resultados obtenidos. La escala visual análoga del dolor, aplicada a las 12 horas en los pacientes sin otra cirugía asociada, fue entre 1 y 2 . No fue necesario agregar otros medicamentos al esquema establecido en las que se realizó TVT-Secur sólo ni en las con otra cirugía asociada.

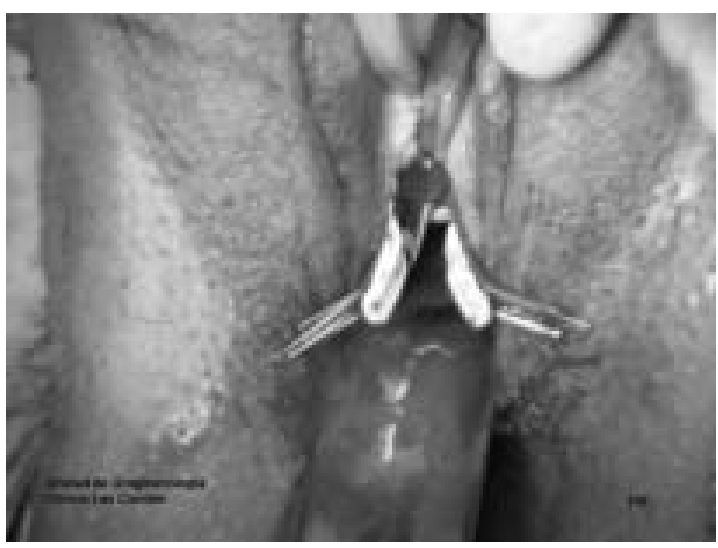

Figura 6. Ajuste de malla suburetral por medio de tijera Metzembaum. 


\section{DISCUSIÓN}

Durante las últimas dos décadas hemos asistido al nacimiento de un gran número de técnicas quirúrgicas en uroginecología (14). Esto ha sido principalmente gracias a la mejor comprensión de la anatomía y fisiología del suelo pélvico, y la aparición de mallas fabricadas con las características apropiadas (15).

La primera generación de cintas suburetrales fue el TVT, de ubicación retropúbica. Su eficacia está probada con un seguimiento de más de 7 años, siendo hoy el estándar dorado en la corrección quirúrgica de la IOE. Sin embargo, el paso retropúbico de las agujas, tiene el riesgo de perforar la vejiga, complicación que generalmente es fácil de solucionar y no deja secuelas. Pero, no debemos olvidar que existe también el riesgo de perforar vísceras abdominales.

EI TOT y TVT-O son la segunda generación de cintas sub-medio-uretrales. En esta técnica las agujas no pasan por el espacio retropúbico, por lo que es menor la probabilidad de provocar lesiones vesicales.

Con el TVT-Secur, asistimos al nacimiento de la tercera generación de cintas. Este sistema posee como principal ventaja, que permite optar a dejar la cinta en una posición igual al TVT o al TVT-O y TOT. Por otro lado, este sistema permite colocar la cinta con una técnica con menor invasión que sus antecesoras, ya que la penetración en el espacio parauretral es sólo de 2 a 2,5 centímetros, lo que también reduce la posibilidad de complicaciones, como la perforación de órganos.

La cinta que se deja in situ es sólo de 8 centímetros, mientras que en las técnicas de primera y segunda generación son 20 centímetros. La reducción del volumen de cuerpo extraño que se introduce, permite obtener el mismo efecto de una cinta sub-medio-uretral. El estudio pre-clínico publicado por Rezapour y cols (16), en 2006, demostró que esta cinta permitía mantener una fuerza diez veces mayor que la tensión sostenida por la fascia. Además, los estudios de esta cinta permitieron comprobar que esta fuerza se mantiene, evitando una posible elongación en el tiempo. Las fuerzas se midieron a 1,2,4 y 12 semanas. Este tópico es muy importante, ya que se trata de una cinta de sólo 8 centímetros, es decir un tercio más corta que sus antecesores, dónde no existe la prolongación lateral que sirve de anclaje. Esto teóricamente podría interpretarse como una posible causa de una menor posibilidad de mantención en su lugar (sub-medio-uretral) y de tensión. La fuerza de fijación de este verdadero sándwich de polipropileno rodeado de PDS (Ethisorb), es mayor que la que logra el polipropileno sólo. En resumen, se comprueba que la fuerza de fijación de la cinta sería equivalente al TVT tradicional.

La malla que utiliza este sistema es la de polipropileno con las características que comprobadamente reducen la posibilidad de complicaciones en las cirugías uroginecológicas. Se trata de una malla clasificada como tipo I en los materiales protésicos (17), que al ser macroporo permite el pasaje de macrófagos en caso de que alguna bacteria llegue hasta la malla (15). Por otro lado, al ser macroporo, permitirá la incorporación de los diferentes elementos que permitirán la tan ansiada biointegración. Al tratarse de mallas monofilamentos, evita que queden espacios entre ellos, en que no penetren los macrófagos (18).

Este sistema presenta claras ventajas sobre sus antecesores, ya que al no requerir agujas para pasar la cinta, posee menos probabilidad de daño de vasos sanguíneos, nervios o perforar la

\section{Tabla II}

\section{PRINCIPALES VENTAJAS DEL NUEVO SISTEMA TVT-SECUR}

- Queda menos cinta en la paciente $(8 \mathrm{~cm}$ vs $20 \mathrm{~cm}$ ).

- No requiere agujas para el paso de la cinta.

- Menos invasión al requerir sólo una zona de apertura de la mucosa vaginal, disección sólo de 2 a 2,5 cm parauretral.

- Permite reposicionar la cinta y ajustarla si es necesario, antes de cerrar la mucosa vaginal.

- Minimiza la posibilidad de perforación de vejiga.

- Menor posibilidad de daño de vasos, nervios y órganos abdomino-pélvicos.

- Mayor versatilidad, ya que permite colocar la cinta en "U" o en "V" (Hammock)

- Al posicionar la cinta en "U" como TVT:

no requiere pasar por el espacio retropúbico.

no requiere salida de la cinta por piel.

- Al posicionar la cinta en "V" como en las técnicas transobturadoras: no requiere salida de la cinta por piel 
vejiga o los órganos de cavidad pélvica y abdominal; además, no requiere puntos de salida de la cinta por la piel, una vez puesta la cinta en posición sub-medio-uretral, se puede reposicionar, si es necesario, antes de cerrar la mucosa vaginal (Tabla II).

Este trabajo es la primera experiencia nacional en la corrección quirúrgica de la IOE, mediante el nuevo dispositivo TVT-Secur, y el análisis preliminar nos permite observar las ventajas que tiene sobre sus antecesores. El seguimiento a largo plazo, que hemos iniciado al igual que con las otras técnicas, nos permitirá comparar la mantención de los buenos resultados en el tiempo.

\section{CONCLUSIONES}

El dispositivo TVT-Secur es una nueva herramienta factible, segura y eficaz en la corrección quirúrgica de la IOE. Permite realizar una cirugía con técnica de cinta suburetral en su tercio medio, con todas las ventajas de la mínima invasión. Es un sistema de cinta de tercera generación, con menor invasión que las técnicas antecesoras, requiere solo una apertura de la mucosa suburetral, sin puntos de salida por piel, dejando sólo 8 centímetros de cinta. Permite colocar la cinta en "U" como en la técnica de TVT o en "V" como las técnicas transobturadoras.

\section{BIBLIOGRAFÍA}

1. Ulmsten U, Henriksson L, Johnson P, Varhos G. An ambulatory surgical procedure under local anesthesia for treatment of female urinary incontinence. Int Urogynecol J Pelvic Floor Dysfunct 1996;7(2):81-5.

2. Rezapour M, Ulmsten U. Tension-free vaginal tape (TVT) in women with mixed urinary incontinence -a long-term follow-up. Int J Urogynecol J Pelvic Floor Dysfunct 2001;12 suppl:15-8.

3. Solà Dalenz V, Pardo Schanz J, Ricci Arriola P, et al. TVT associated with other gynecological operations in the same procedure: results and complications. Arch Esp Urol 2005;58(10):983-8.

4. Delorme E. Transobturator urethral suspension: miniinvasive procedure in the treatment of stress urinary incontinence in women. Prog Urol 2001;11(6):130613.
5. Delorme E, Droupy S, De Tayrac R, Delmas V. Transobturator tape (Uratape). A new minimally invasive method in the treatment of urinary incontinence in women. Prog Urol 2003;13(4):656-9.

6. Pardo Schanz J, Ricci Arriola P, Solà Dalenz V, et al. Trans-obturator-tape (TOT) for the surgical repair of stress urinary incontinence: our experience. Arch Esp Urol 2006;59(3):225-32.

7. De Leval J. Novel surgical technique for the treatment of female stress urinary incontinence: transobturator vaginal tape inside-out. Eur Urol 2003;44(6): 724-30.

8. Solà Dalenz V, Pardo Schanz J, Ricci Arriola P, et al. Minimal invasive surgery in female urinary incontinence: TVT-O. Actas Urol Esp 2006;30(1):61-6.

9. Solà V, Pardo J, Ricci P. Cirugía mínimamente invasiva en el tratamiento de la incontinencia urinaria femenina de esfuerzo: TVT-O. Rev ChIL OBStET GINECOL 2006;71(1):3-9.

10. Martan A, Masata J, Svabik K. TVT SECUR systemtension-free support of the urethra in women suffering from stress urinary incontinence-technique and initial experience. Ceska Gynekol 2007;72(1): 42-9.

11. McGuire EJ, Fitzpatrick CC, Wan J, et al. Clinical assessment of urethral sphincter function. J Urol 1993;150(5 Pt 1):1452-4.

12. Debodinance $P$, Cosson $M$, Collinet $P$, et al. Synthetic meshes for transvaginal surgical cure of genital prolapse: evaluation in 2005. J Gynecol Obstet Biol Reprod (Paris) 2006;35(5 Pt 1):429-54.

13. Pardo J, Solà V, Ricci $P$, et al. Colpoperineoplasty in women with a sensation of wide vagina. Acta Obstet Gynecol Scand 2006;85(9):1125-7.

14. Martan A. New options in reconstructive pelvic floor surgery and surgery in urogynecology. Ceska Gynekol 2006;71(6):455-63.

15. Solà V, Pardo J, Ricci P, Guiloff E. Mallas protésicas en el tratamiento quirúrgico del prolapso genital en la mujer. Rev Med Clin Condes. 2005;16(3):168-79.

16. Rezapour M, Novara G, Meier PA, et al. A 3-month preclinical trial to assess the performance of a new TVT-like mesh (TVTx) in a sheep model. Int Urogynecol J Pelvic Floor Dysfunct 2007;18(2):183-7.

17. Birch C, Fynes MM. The role of synthetic and biological prostheses in reconstructive pelvic floor surgery. Curr Opin Obstet Gynecol 2002;14(5):527-35.

18. Solà V, Pardo J, Ricci P, Guiloff E. Tension free monofilament macropore polypropilene mesh (Gynemesh PS) in female genital prolapse repair. Int Braz J Urol 2006;32(4):410-4;discusión 415. 